\title{
Escola médica e Sistema Único de Saúde (SUS): criação do curso de medicina da Universidade Federal de São Carlos, SP, Brasil (UFSCar) sob perspectiva de docentes e estudantes*
}

Maristela Schiabel Adler(a)

Dante Marcello Claramonte Gallian ${ }^{(\text {b) }}$

Adler MS, Gallian DMC. Medical school and Brazilian National Health System (SUS): implantation of the Federal University of Sao Carlos (UFSCar) medical school from the perspective of teachers and students. Interface (Botucatu). 2018; 22(64):237-49.

The Federal University of Sao Carlos (UFSCar) launched in 2006 the UFSCar Medical School, with constructivist, competency curriculum and practice based learning, interweaved in the SUS structure. The objective of the present study is to analyze the perceptions of teachers and students from the first group of graduates about their experience usingthe methodology called Life Oral History. Despite reports of infrastructure deficiencies, the narratives suggest that the curriculum favored the development of competencies such as search capability, critical and reflective thinking and selfmanaged learning. The fact of having practice integrated into the SUS, especially within the Family Health Units, improved intellective, relational and affective dimensions of patient care, favoring professional autonomy.

Keywords: Undergraduate medical course. Competency-based education. Problembased learning. Brazilian National Health System.

\author{
A Universidade Federal de São Carlos \\ (UFSCar) implantou seu curso de Medicina \\ em 2006, com currículo baseado em \\ competências e aprendizado prático \\ integrado ao SUS. O objetivo deste \\ trabalho é apresentar uma pesquisa com \\ docentes e graduandos da Primeira Turma \\ da Medicina-UFSCar, realizada por meio da \\ metodologia da História Oral de Vida. As \\ narrativas indicam que o currículo favoreceu \\ o desenvolvimento de competências \\ como: capacidade de busca, pensamento \\ crítico-reflexivo e autogerenciamento \\ do aprendizado, apesar de relatos sobre \\ deficiências em infraestrutura. A prática \\ integrada ao SUS, especialmente nas \\ Unidades de Saúde da Família, aprimorou as \\ dimensões intelectivas, relacionais e afetivas \\ do cuidado, favorecendo a autonomia \\ profissional. \\ Palavras-chave: Ensino de graduação \\ em medicina. Educação baseada em \\ competências. Aprendizagem baseada em \\ problemas. Sistema Único de Saúde.

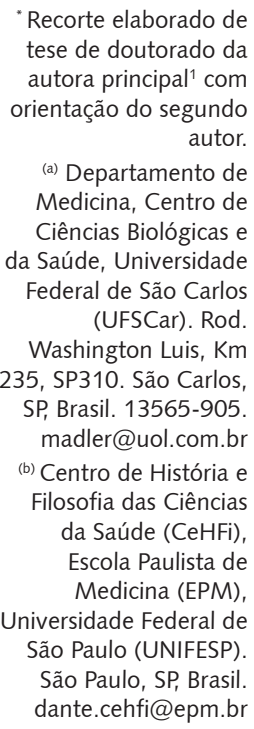

"Recorte elaborado de tese de doutorado da autora principal ${ }^{1} \mathrm{com}$ orientação do segundo

(a) Departamento de Medicina, Centro de Ciências Biológicas e Federal de São Carlos (UFSCar). Rod. Washington Luis, Km SP, Brasil. 13565-905. madler@uol.com.br (b) Centro de História e Filosofia das Ciências da Saúde (CeHFi), Escola Paulista de Medicina (EPM), São Paulo (UNIFESP). São Paulo, SP, Brasil. dante.cehfi@epm.br \\ autor. \\ da Saúde, Universidade \\ 235, SP310. São Carlos, \\ Universidade Federal de
}




\section{Introdução}

Propostas contemporâneas de modelos curriculares para o cuidado em saúde incentivaram mudanças, oficializadas nas Diretrizes Curriculares Nacionais $(D C N)^{2}$ da graduação em Medicina, que privilegiaram uma formação generalista, humanista, crítica, reflexiva e ética, capaz de ações de promoção, prevenção, recuperação e reabilitação da saúde, visando a uma assistência integral ao ser humano, com responsabilidade social.

Currículos estruturados no Problem-Based Learning (PBL) introduziram o estudo de SituaçõesProblema como núcleo do ensino da Medicina nas Faculdades de Medicina de Marília (FAMEMA) e de Londrina (UEL) ${ }^{3-5}$.

A escola de Medicina da Universidade Federal de Santa Catarina, em sua reforma curricular de 2003, optou por um modelo curricular com estrutura modular, sob eixo didático-pedagógico de orientação construtivista, integrando o ciclo básico ao profissionalizante ${ }^{6}$. Já a Faculdade de Medicina da Universidade de São Paulo reformulou seu currículo, aproximando-o das DCN e do SUS, mantendo sua metodologia de ensino tradicional.

Em busca de novos caminhos para o ensino médico, a Universidade Federal de São Carlos (UFSCar) inaugurou em 2006 sua graduação, cujo Projeto Político Pedagógico (PPP) fundamentou-se em três pressupostos: currículo orientado por competência, abordagem educacional construtivista e integração teoria-prática voltada para o SUS.

Em um estudo proposto como tese de doutorado ${ }^{1}$, foi apresentada a trajetória de construção da Medicina-UFSCar sob a perspectiva de nove docentes e oito estudantes que participaram do processo entre os anos de 2006 e 2011. Desse modo, o objetivo deste artigo é o de apresentar e discutir os temas mais relevantes que emergiram nas narrativas dos participantes do estudo.

\section{Metodologia}

A metodologia utilizada no estudo foi a História Oral de Vida (H.O.), abordagem qualitativa que se fundamenta na apreensão de narrativas de experiências de um grupo sobre determinados aspectos de suas vidas. É uma forma específica de abordar a realidade, recolher testemunhos, promover análises de processos sociais do presente, e facilitar o conhecimento do meio proposto no estudo que permite ampla compreensão do individual e do coletivo em determinado ponto da história9-11.

A H.O. foi realizada com a participação individual de nove docentes e oito formandos, ingressantes na Medicina-UFSCar no ano de 2006. Tal metodologia foi escolhida por propiciar o acesso à dimensão das experiências dos sujeitos envolvidos no processo. Os colaboradores do estudo foram designados por nomes fictícios para preservar suas identidades.

Para a análise das narrativas, foi utilizado o método de imersão/cristalização ${ }^{12,13}$, a abordagem analítica de caráter fenomenológico ${ }^{14} \mathrm{e}$ o processo analítico hermenêutico ${ }^{15,16}$, que teve como finalidade buscar, nos fenômenos descritos, significados das percepções dos entrevistados sobre suas experiências vividas e suas relações com o mundo no qual foram inseridos.

\section{Resultados e discussão}

\section{O início da Escola}

A UFSCar planejou sua formação médica com base nas DNC de 2001², cujo currículo contemplou: a formação por competências, as metodologias ativas de ensino-aprendizagem, e a parceria entre a Universidade e a Rede de Saúde Municipal, integrando alunos, docentes e preceptores do SUS, de primeiro a sexto ano.

"O Curso da UFSCar nasce diferente, já nasce numa parceria" (Professor Jorge). 
O PPP da Medicina-UFSCar propôs mudanças de paradigmas estabelecidos, como: a extinção de aulas expositivas e a formação profissional por competências sob uma visão ampliada, humanizada, ética, social e articulada com equipes interdisciplinares ${ }^{2,8}$.

Também propôs mudanças na concepção de cuidado em saúde, com o paciente no centro das ações ${ }^{17-19}$. A integralidade do cuidado foi definida como eixo central das atividades curriculares, visando a uma formação generalista, com foco na prevenção e promoção à saúde e participação ativa na gestão, com enfoque no SUS ${ }^{8}$.

Segundo a professora Júlia, "a estrutura não tinha sido bem preparada para a recepção do Curso", o que resultou na capacitação e construção das atividades pedagógicas simultaneamente à realização do Curso.

"Esse início foi muito complicado e, ao mesmo tempo, um aprender fazendo, também difícil porque não havia mais tempo para discutirmos. Tínhamos alunos". (professor Mário)

O reduzido tempo para capacitação ${ }^{20,21}$, no entender dos colaboradores, prejudicou o Curso nos primeiros anos, o que, somado à falta de uma Matriz Curricular, resultou em instabilidades na aprendizagem. Porém, após o primeiro impacto, professores e estudantes se identificaram com as atividades didáticas, que permitiram o desenvolvimento do processo crítico-reflexivo e de habilidades cognitivas, afetivas e relacionais ${ }^{22,23}$.

Ao compararem o ensino da Medicina UFSCar e o ensino tradicional, os alunos deram preferência ao primeiro. "Fazia mais sentido para mim... A Faculdade dessa maneira, pedagogicamente, me fez estudar mais", disse o estudante Luciano.

\section{O referencial metodológico do Curso}

A abordagem educacional construtivista foi escolhida como norteadora do currículo, estimulando o estudante a aprender a aprender (aprender a ser, aprender a fazer, aprender a viver juntos e aprender a conhecer) $)^{2,8,24}$.

Esse currículo colocou o aluno no papel central do conhecimento por meio de metodologias ativas de ensino-aprendizagem ${ }^{25-27}$, no caso, as Situações Problema (SPs) e as Estações de Simulação da Prática Profissional 28,29 .

O estudo de situações clínicas hipotéticas, apresentadas em papel (SPs), cujo eixo teórico metodológico fundamenta-se no PBL, permitiu uma aprendizagem cognitiva em espiral. Contudo, o trabalho interativo foi fator de estranhamento para todos. Os estudantes, não habituados à busca e construção de conhecimentos para as atividades, relataram dificuldades de aprendizagem com o método 22,30 .

A metodologia retirou os estudantes de suas zonas de conforto, pediu uma aprendizagem críticoreflexiva e os responsabilizou frente à elaboração individual e coletiva do saber, com o docente no papel de facilitador ${ }^{8,25,31}$, provocando inseguranças frente ao aprendizado. Em contrapartida, tais atividades possibilitaram a aprendizagem integrada de dimensões biológica, psicológica e social ${ }^{8,29}$.

Segundo os colaboradores, essa aprendizagem foi heterogênea, devido à falta de afinidade dos facilitadores com a metodologia e pelo tipo de capacitação ofertada, que implicava a não interferência destes em discussões das SPs. Na opinião do aluno Vicente, isso era um "PBL mais radical", o que contrasta com a forma original do PBL, em que o facilitador tem o papel de aperfeiçoar as habilidades do pensamento, ajudando o estudante a modelar a ordem superior das ideias com perguntas que aprofundem os saberes, e apoiar o desenvolvimento em sua lógica cognitiva ${ }^{32,33}$. Almeida e Batista ${ }^{32}$ afirmam que o PBL tem, em sua essência, o "desempenho do docente como sujeito da educação e orientador do processo de ensino-aprendizagem" 32 (p.200).

Nas Estações de Simulação, a prática clínica foi trabalhada em duplas de estudantes, com situações clínicas construídas pelos docentes e encenadas por pacientes-atores. Atividade que possibilitou o desenvolvimento das habilidades profissionais em ambiente controlado, onde as lacunas de aprendizagem e erros atitudinais puderam ser corrigidos in loco $0^{28,34,35}$. 
Para os entrevistados, o resultado dessas práticas pedagógicas aponta para um aprendizado desigual, porém mais significativo por estruturar-se no estudo autogerenciado e em experiências individuais ${ }^{36,37}$. Por basear-se na construção do conhecimento individual, a aprendizagem significativa só se efetiva, segundo David Ausubel ${ }^{38}$, sobre um conhecimento anterior já consolidado, o que sempre será inerente às experiências individuais de cada aluno ${ }^{28,39,40}$.

\section{No SUS, o ensino em construção}

Para a aprendizagem no SUS, a UFSCar fez uma parceria com o Poder Municipal, que se responsabilizou por fornecer os recursos estruturais, materiais e humanos, cujos cenários necessitavam de grandes investimentos e era integrado por equipes que tinham dificuldades para desenvolver a assistência integrada. Segundo os docentes, a proposta da Escola foi de qualificar o serviço, o que já havia acontecido em Marília e Londrina ${ }^{3,41}$.

No planejamento inicial, o Curso trabalharia com três preceptores para cada docente. A preceptoria era um dos pilares de sustentação do PPP, com o preceptor estando junto aos estudantes na maior parte do tempo. Porém, a falta deste profissional na Rede, sua alta rotatividade no serviço, e responsabilidade em cumprir sua carga horária frente ao SUS (além de orientar os estudantes), contribuiu para a inadequação dessa diretriz do Curso.

“Em função da sobrecarga de trabalho, os preceptores têm dificuldades em recebê-los [estudantes]. Falta investimento financeiro adequado que possa trazer profissionais para a cidade". (Professora Júlia)

No início, a Prefeitura fez melhoramentos nas Unidades de Saúde, com boa repercussão para o aprendizado, mas, ao longo dos anos, reduziu a adequação e criação de outras Unidades, limitando a oferta de cenários para a prática, e a parceria se mostrou aquém do planejado. A Universidade não obteve autonomia na Rede de Saúde para efetuar as mudanças necessárias à implantação adequada do Curso.

"O parceiro que é justamente a Prefeitura, não proveu isso. Hoje, não temos nem as Unidades de Saúde da Família (que seria o básico) e nem as Unidades Básicas prometidas. Não há cenários, nem em quantidade suficiente, nem com qualidade". (Professora Rita)

A infraestrutura deficiente ${ }^{42}$ e mudanças de políticas locais de saúde prejudicaram o aprendizado prático. "No momento em que o Curso se vincula à instabilidade política, se torna instável e isto não pode acontecer" (Estudante Vicente), e a Escola se viu frente a obstáculos para a incorporação da graduação pelo Serviço Público de Saúde Municipal.

“É um gargalo sem solução a curto prazo. Um problema que persistirá por muitos anos porque o Curso continua sendo dependente da atuação do governo local, principalmente em relação à utilização do Sistema de Saúde da cidade..." (Estudante Antônio)

Com menos de cinquenta docentes e cinquenta preceptores (com estimativa para cento e cinquenta), os professores ficaram sobrecarregados, com encargos da graduação, exigências de pesquisa e publicações, sem tempo para capacitação, educação permanente, planejamento de atividades curriculares e matriciamento junto às Unidades do $S U S^{43}$, o que se refletiu na diminuição do potencial de ensino-aprendizagem do Curso.

“Um curso de graduação em Medicina que precisa ter um corpo docente extremamente compartilhado, empenhado em implementar o currículo diariamente, no molde onde a pesquisa vem primeiro que a graduação, vai dar problema!" (Estudante Augusto) 
Segundo as narrativas, apesar dos percalços, os alunos foram inseridos, de primeiro a sexto ano, nas atividades práticas do SUS, em Unidades Básicas de Saúde (UBSs) e Unidade de Saúde da Família (USFs). Nas USFs, em visitas domiciliares de rotina, construíram uma coorte a partir de famílias que acompanharam ao longo dos anos, aprendendo a visão individual e coletiva do cuidado.

O enfoque do cuidado integral, centrado no paciente e de uma prática médica humanizada, exemplificado por docentes e preceptores, foi avaliado como positivo pelas narrativas dos estudantes e permitiu o desenvolvimento de habilidades para o mundo do trabalho. Isso em contraponto ao que acontece em outros currículos, em que a excessiva importância confiada à dimensão biológica e técnica do conhecimento faz com que o estudante infira que tais dimensões sejam suficientes para a formação profissional25,44.

A inserção longitudinal na prática do SUS ${ }^{4,23,45}$, seja no acompanhamento de famílias ou em atividades de gestão, contribuiu: para a sedimentação de conhecimentos, para a percepção da realidade na Saúde Pública, da responsabilidade profissional e social ${ }^{46}$, e estimulou a participação ativa dos estudantes junto ao SUS e à comunidade ${ }^{46-49}$. Segundo o estudante Luciano, eles aprenderam a "lidar com a população de maneiras mais ativas". O enfoque na Atenção Primária influenciou a mudança do perfil profissional, permitindo, ao estudante, a gestão dos problemas prementes da população.

"Tivemos muitas oportunidades de discutir sobre nosso papel na sociedade e na coresponsabilização junto ao paciente, enxergá-lo como um todo e dentro de uma sociedade repleta de desavenças..." (Estudante Vicente)

As habilidades éticas e humanísticas tiveram as ferramentas de desenvolvimento na valorização da escuta do paciente e na sua contextualização, segundo os docentes Jorge e Clara. Para os estudantes, esse foi outro ponto forte do currículo, exemplificado nas USFs, onde o responsabilizar-se pelas famílias possibilitou: a criação do vínculo, a percepção diferenciada do outro, o respeito pelo serviço, e menor grau de defesa na relação médico-paciente. O modelo horizontalizado de poder preconizado pelo PPP, segundo a professora Cora, também contribuiu para o aprendizado ${ }^{50}$.

“Tínhamos que ficar responsáveis por algumas famílias no estágio. Conheci a fundo estas pessoas... Mais do que isso, foi a questão humana mesmo. Foi boa essa prática... A parte humana foi o que falou mais alto nesses primeiros anos, pelo fato da prática ter se iniciado mais cedo... Lembro com saudade de muitos pacientes que tive, alguns faleceram quando eu estava no quarto ano. Ficou um sentimento bom. A questão humana da formação como um todo, temos bastante e levaremos muito conosco". (Estudante Ricardo)

Segundo o docente Hélio, o enfrentamento de adversidades e a busca de soluções, nos cenários do SUS, aumentou a capacidade resolutiva dos alunos ${ }^{27,49}$.

As discussões problematizadas na realidade do SUS possibilitaram aprendizagem distinta aos estudantes. Considera-se aqui que problematizar é uma experiência dotada de sentido, segundo Bondia $^{12}$, onde se produz não apenas o conhecimento, mas se dá, ao aluno, a possibilidade de ser o 'sujeito da experiência', capaz de unir o conhecimento à vida humana e sedimentar o que viveu ${ }^{12,40}$.

"No futuro, no exercício da profissão, o aprendizado vai nos ajudar a influenciar e modificar o ambiente de trabalho, a ser mais crítico, saber trabalhar em grupo, saber organizar, parar, pensar e resolver os problemas". (Estudante Ricardo)

No SUS de São Carlos, as Unidades de Saúde da Família mostraram-se as mais organizadas, integradas e responsivas aos anseios de formação do Curso. Foram cenários propícios à construção do cuidado integral, interprofissional e humanista ${ }^{51}$. Já nas UBSs, "um desastre: modelo de Pronto Atendimento"; segundo a professora Sofia, o modelo utilitarista de queixa-conduta não permitiu a aprendizagem do cuidado integral de forma adequada e expôs o estudante à velha hegemonia da 
Medicina Diagnóstica e à informação verticalizada ${ }^{45,52}$; e contribuiu para a baixa resolutividade do Sistema de Saúde, tornando o cuidado em saúde insatisfatório e cada vez mais oneroso $20,25,48,53$.

Os estudantes consideraram a aprendizagem para a atuação na Atenção Primária, com foco na integralidade do cuidado à saúde e das necessidades sociais da população assistida, um dos pontos fortes do currículo. E se julgaram preparados para o exercício da profissão nesse nível de atenção.

“Não tenho medo de fazer Atenção Básica... Vou para um USF tranquilamente! Não há problemas. Os pacientes que estão lá, têm problemas que vi ao longo os seis anos de formação e se tiver dúvida, serei capaz de buscar informações que possibilitem a tomada de decisão. Tive a formação. A graduação me deu isso e foi fundamental". (Estudante Augusto)

No decorrer dos seis anos, os desacordos políticos resultaram em um número de preceptores e Unidades aquém do necessário para a aprendizagem individualizada, o que foi agravado pelo pequeno número de docentes presentes na prática, dificultando a qualificação do serviço pela Academia ${ }^{8,43,49}$.

A Academia junto ao Serviço de Saúde pode auxiliar integrando saberes, e, na opinião de Laura Feuewerker ${ }^{25}$, o fato de uma faculdade instituir atividades no SUS não correspondente necessariamente a um aprendizado adequado e à busca de um modelo diferente do hospitalocêntrico que envolva a Rede de Serviços Públicos, mostra-se tarefa de enorme complexidade ${ }^{20,54}$.

Troncon ${ }^{20}$ nos fala que, para a efetivação do ensino/pesquisa/assistência de qualidade no SUS, as Unidades devem estar organizadas, oferecer um número amplo de ações integradas, inclusive, junto à comunidade. O número de trabalhadores deve ser suficiente, capacitado e motivado para a supervisão dos alunos. O contato com os estudantes deve ficar a cargo de profissionais que sabidamente detenham um perfil atitudinal adequado e funcionem como modelos apropriados, no qual os estudantes se espelhem. O número de pacientes deve ser o suficiente para as atividades educacionais. E, por fim, são necessários vínculos estáveis entre a Universidade e o Serviço de Saúde que mantenham canais abertos entre a graduação e a comunidade, para o desenvolvimento do ensino nesses cenários.

Entretanto, em nosso país, a integração entre as Escolas Médicas, os Serviços Públicos de Saúde e setores governamentais é deficiente, e o SUS apresenta dificuldades de toda ordem para corresponder às suas diretrizes ${ }^{48,49,51}$. A baixa resolutividade e alto percentual de encaminhamentos, os baixos salários, a falta de capacitação e o excesso de pacientes diminuem a possibilidade de aprendizagem no SUS $21,42,55$.

\section{O Internato}

Após grande movimentação dos estudantes, com duas paralisações que reivindicavam melhores condições de ensino, o Internato, programado para acontecer em São Carlos, foi transferido para Américo Brasiliense, Bauru, Limeira e Diadema, devido ao não cumprimento do prazo de entrega do Hospital Universitário (previsto, inicialmente, para 2010). Com isso, perdeu-se o referencial pedagógico metodológico do Curso: o ensino-aprendizagem centrado no estudante, a autonomia para construção do conhecimento, o desenvolvimento das habilidades crítico-reflexivas, e a integralidade do cuidado à saúde ${ }^{8}$. Os estudantes habituados a esse movimento tiveram dificuldade na adaptação a distintas formas de pensar e agir de outras Escolas, o que lhes causou sofrimento (por não reconhecerem como verdadeiros diferentes processos de ensino) e amadurecimento (por terem de se adaptar a outras realidades, onde o processo crítico-reflexivo não fazia parte da rotina de docentes e estudantes).

"Em alguns lugares se pede Tomografia Computadorizada de manhã e à tarde! Estranho! O que é isto? Isto não existe! Não deve ser assim a formação. Eles têm uma deformação". (Estudante Augusto)

Nos estágios, muitas vezes não eram bem recebidos. Habituados a questionar (habilidade valorizada pelo Curso), foram hostilizados por alguns docentes e residentes de outros serviços ${ }^{50,56}$. 
O desconhecimento da metodologia na qual os estudantes estavam sendo preparados pode ter sido um complicador dessa relação. A desumanização do processo de treinamento nas Escolas Médicas também, possivelmente, contribuiu para a falta de acolhimento dos jovens, o que, em parte, desestimulou-os para os estudos ${ }^{50,56,57}$.

Outro ponto complicador para os estudantes foi trabalharem com uma abordagem ampliada do doente em Planos Terapêuticos Singulares. Desde a anamnese até a conduta compartilhada, o enfoque dado pelo Curso sempre foi a integralidade do cuidado, com discussões e ações em equipes interdisciplinares. "Mas o mundo lá fora não mudou", afirmou o estudante Augusto ao relatar que fora do ambiente protegido da UFSCar não havia semiologia com história ampliada.

O trabalho realizado junto aos alunos, focado na individualização do aprendizado e necessidades de saúde do paciente, auxiliou na compreensão e sedimentação do modelo biopsicossocial e relacional proposto pelo currículo que os estudantes levaram para o Internato. Ao se depararem com o modelo biomédico de queixa-conduta, entenderam ser o modelo da UFSCar mais adequado para a completude do cuidado e para o exercício profissional.

“Esta é uma preocupação muito intensa no nosso Curso, de ter orelhas grandes para escutar bastante e olhos bem abertos para enxergar bem. O conhecer o outro... Não só conhecer, é reconhecer, pactuar, construir para ajudar de alguma maneira no jeito que ele leva sua vida". (Professor Jorge)

Ao final dos seis anos, os alunos evidenciaram cansaço e desesperança na implantação desse currículo. A insegurança frente a tantas mudanças tornou-se superlativa e parte deles recorreu a tratamento psiquiátrico para concluir o Curso $0^{1,57}$. Em seus relatos, observou-se um nítido esgotamento das relações com a Universidade, com a Escola e interpessoais.

Apesar dos percalços, o que emerge das narrativas dos estudantes como resultante das atividades curriculares é a visão ampliada do cuidado, embasada em um modelo de conduta compartilhada, interprofissional, ética e humanizada.

"[...] aprender a olhar o indivíduo, tentar entender suas correlações e junto com ele achar uma solução, obter resultados melhores na terapêutica, saber quais são os limites do momento e qual nosso papel ali... Compreender como a equipe lidou com o caso". (Estudante Vicente)

Entretanto, no Internato, os estudantes referem que o ensino foi insuficiente e o envolvimento com o SUS, aquém do desejado. Pelo planejamento inicial, no Hospital Universitário, eles continuariam o aprendizado do cuidado ampliado, desenvolvendo atividades e integrando os três níveis de atenção. Mas, com a pulverização do estágio, o "Curso não pode manter o controle sobre a qualidade do ensino", comentou a professora Cora. Mesmo assim, a formação proporcionada nos anos anteriores mostrou-se suficiente para que os alunos desenvolvessem uma grande capacidade de enfrentamento, utilizando habilidades crítico-reflexivas para a resolução de problemas.

\section{Ao final dos seis anos}

O Curso de Medicina da UFSCar estabeleceu-se como parceiro do SUS, apesar das dificuldades enfrentadas. Para os docentes, as questões políticas locais e o pouco entendimento (de órgãos gestores e profissionais do SUS) do cuidado integral em saúde foram os maiores obstáculos enfrentados para integrar a Academia à Rede.

$\mathrm{Na}$ observação dos professores, os estudantes mostraram amadurecimento, comprometimento e domínio das habilidades profissionais para a Atenção Primária, o que foi atribuído: à metodologia, às dificuldades enfrentadas, à responsabilização frente à implantação do currículo, e por terem de ser exemplo para as turmas subsequentes.

A complexidade do PPP obrigou a inúmeras mudanças de rumo. "Hoje, não temos o mesmo Curso do início, temos um outro, o que foi possível" (Professora Cora). 
Esse currículo, cuja formação se vinculou ao SUS de forma significativa e onde a conduta compartilhada definiu as ações multiprofissionais ${ }^{46}$, aponta que currículos transformadores, como definiu Lampert" ${ }^{47}$, potencializam a formação. "A potência do Curso está dada... A primeira turma sai e mostra isto" (professora Júlia).

A dedicação dos estudantes foi importante para a implantação. "A primeira turma foi parceira durante os seis anos exigindo, brigando por melhores condições de ensino" (Professora Magda).

Atualmente, o Curso continua em transformação, com o Internato acontecendo em São Carlos, no Hospital Universitário (administrado pela Empresa Brasileira de Serviços Hospitalares - EBSERH), na Unidade de Saúde Escola-UFSCar e na Santa Casa da cidade ${ }^{58,59}$. Para suprir a falta de preceptores da prática profissional no SUS, a solução encontrada foi a alocação de docentes nessa atividade. Segundo a Professora Sofia, "O que pensamos fazer em quatro anos, talvez façamos em quinze!", com a necessidade de se repensarem os pontos frágeis do Curso.

\section{Considerações finais}

Este artigo apresentou as percepções de alunos e docentes sobre o processo de formação médica da UFSCar, em que a metodologia da História Oral possibilitou a abordagem de experiências e vivências da primeira geração da Escola.

Os pesquisadores entendem que a importância desse trabalho está na reflexão que traz sobre projetos educacionais em processo de construção e/ou reformulação, de modo que os achados aqui relatados poderão auxiliar na discussão sobre novos caminhos para os currículos médicos.

O Curso ofereceu um currículo orientado por competências, sob abordagem educacional construtivista, com base em PBL e metodologias ativas de ensino-aprendizagem que permitiram centrar as atividades pedagógicas no aluno.

A literatura aponta que definir o PBL como a linha mestra de currículos mostra-se um caminho possíve $\left.\right|^{36,37,45}$. No entendimento dos pesquisadores, associar o PBL a metodologias ativas permitiu a potencialização do aprendizado $29,34,45$. Porém, a insegurança e heterogeneidade geradas pelo processo pedem o acréscimo de abordagens pedagógico-metodológicas que ajudem na sedimentação das aprendizagens e proporcionem segurança aos estudantes ${ }^{1,60-62}$.

O início das atividades do Curso, antes da capacitação dos docentes e preceptores, pareceu um erro de planejamento e não pode ser exemplo para implantações de futuras escolas.

O número de cinquenta docentes e cinquenta preceptores, considerado insuficiente pelos colaboradores para o desenvolvimento das atividades curriculares, reduziu o potencial de aprendizagem.

Os desacordos políticos e as dificuldades de uma cogestão com a Universidade da prática profissional no SUS foram obstáculos para a aprendizagem prática desejada pela Escola. Os cenários foram insuficientes. O subfinanciamento do SUS não possibilitou infraestrutura adequada ao ensino. O apoio matricial, sob responsabilidade da Escola, bem como a capacitação dos profissionais da Rede para acompanhamento dos estudantes foram acanhados.

Apesar das dificuldades, a vivência nas USFs no acompanhamento longitudinal de famílias e as atividades de gestão permitiu, aos estudantes, a aquisição de competências desejadas pelo Curso. Eles aprenderam a elaborar Planos Terapêuticos Singulares e vivenciaram o comprometimento profissional e social. Aprimoraram dimensões intelectivas, relacionais e afetivas no cuidado ampliado, desenvolvido sob o modelo de conduta compartilhada e interprofissional. Adquiriram autonomia e resolutividade, relatando aptidão satisfatória para o exercício da profissão na Atenção Primária, sob uma compreensão realista do SUS, e criaram vínculos que ajudaram na aprendizagem da humanização do cuidado.

O discurso de inclusão, ética e responsabilização social, segundo os estudantes, foi vivenciado no exercício diário da graduação e funcionou como fator diferenciador da formação.

As atividades práticas no SUS, com enfoque na clínica centrada no paciente ${ }^{18,19}$, influenciaram o desenvolvimento de habilidades humanísticas e na mudança do perfil de formação profissional, comprovando a importância do exemplo positivo dos tutores junto ao aluno ${ }^{20}$. 
Os estágios do Internato, realizados em unidades de ensino não pertencentes à UFSCar, resultaram na perda do referencial pedagógico metodológico do Curso. Porém, os estudantes conservaram as habilidades crítico-reflexivas e a visão do cuidado integral, evidenciando a sedimentação dos conhecimentos adquiridos nos anos anteriores.

A primeira turma relatou insegurança frente ao desenvolvimento de habilidades cognitivas e deficiência de aprendizagem técnica no Curso, o que atribuíram a inúmeras alterações e não realizações de atividades planejadas inicialmente.

A graduação proposta pela Medicina-UFSCar mostrou-se uma alternativa para o caminho do ensino médico, ao possibilitar a experiência de um currículo que prioriza uma formação médica sob competências, enfatizando a Atenção Primária nos cenários do SUS e instigando a reflexão crítica sobre o estudo e vivências tutoradas. É um modelo que pode servir de inspiração para outras Instituições que almejam uma formação voltada para a integralidade do cuidado e para o SUS $8,47,63$.

Docentes e estudantes da primeira turma tiveram um papel vital na estruturação do currículo. Entrar em uma Universidade, deparar-se com um PPP a ser construído, capacitar-se nas metodológicas solicitadas pelo currículo, e corresponder às exigências das tarefas pessoais (graduando ou docente) é, no mínimo, laborioso.

"Participar desse processo de implantação foi um diferencial muito grande na minha vida". (Estudante Vicente)

"Valeu a pena, apesar de tantas dificuldades", foi uma observação que se repetiu nas narrativas. Entretanto, há que se deixar uma pergunta para o futuro: Deve-se ratificar, como virtude, uma formação fundamentada na luta, nas dificuldades e nas carências?

\section{Colaboradores}

Na elaboração do artigo ambos os autores participaram, igualmente, da elaboração, discussão, redação, revisão e aprovação da versão final do trabalho.

\section{Referências}

1. Adler SM. Aprendizado em construção: as vozes da primeira turma da Faculdade de Medicina da Universidade Federal de São Carlos [tese]. São Paulo (SP): Universidade Federal de São Paulo; 2015.

2. Ministério da Educação (BR). Conselho Nacional de Educação. Orientação para as diretrizes curriculares dos cursos de graduação. Diário Oficial da União. 29 Out 2001.

3. Lima VV, Komatsu RS, Padilha RQ. Desafios ao desenvolvimento de um currículo inovador: a experiência da Faculdade de Medicina de Marília. Interface (Botucatu). 2003; 7(12):175-84.

4. Berbel NAN. A problematização e a aprendizagem baseada em problemas: diferentes termos ou diferentes caminhos? Interface (Botucatu).1998; 2(2):139-54. 
5. UEL Prograd. Caderno dos cursos de graduação. Londrina: Universidade Estadual de Londrina, Centro de Ciências da Saúde; 2015.

6. Universidade Federal de Santa Catarina, Centro de Ciências da Saúde, Curso de Graduação em Medicina. Projeto Pedagógico-Curso de Graduação em Medicina. Florianópolis; 2015.

7. Vieira JE, Lopes Júnior A, Basile MA, Martins MA. Identificação das diretrizes curriculares nacionais do curso de graduação em medicina pelos alunos da Universidade de São Paulo. Rev Bras Educ Med. 2003; 27(2):96-103.

8. Medicina UFSCar. Curso de Medicina - Projeto Político Pedagógico [Internet]. 2007 [citado 22 Maio 2014]. Disponível em: http://www.prograd.ufscar.br/projetoped/projeto medicina.pdf.

9. Holanda F, Meihy JCSB. História oral: como fazer, como pensar. São Paulo: Contexto; 2007.

10. Gallian DMC. 75X75: EPM/UNIFESP, uma história, 75 vidas. São Paulo: Unifesp; 2008.

11. Gallian DMC, Holanda F, Meihy JCSB, Silva MRB, Ribeiro SLS, Reginato V. Recortes da memória: lembranças, compromissos e explicações sobre a EPM/UNIFESP na perspectiva da história oral. São Paulo: Unifesp; 2009.

12. Borkan J. Immersion/cristalization. In: Miller WL, Crabtree BF. Doing qualitative research. 2a ed. Thousand Oaks: Sage; 1999.

13. Bondia JL. Notas sobre a experiência e saber de experiência. Rev Bras Educ. 2002; 19:20-8.

14. Devechi CPV, Trevisan AL. Sobre a proximidade do senso comum das pesquisas qualitativas em educação: positividade ou simples decadência? Rev Bras Educ. 2010; 15:148-61.

15. Bruns MAT. A redução fenomenológica em Husserl e a possibilidade de superar impasses entre a subjetividade e a objetividade. In: Bruns MAT, Holanda AF, organizadores. Psicologia e pesquisa fenomenológica: reflexões e perspectivas. São Paulo: Ômega; 2005.

16. Seibt CL. Heidegger: da fenomenologia 'reflexiva' à fenomenologia hermenêutica. Princípios. 2012; 19(31):79-98.

17. Aguilar-da-Silva RH, Perim GL, Abdalla IG, Costa NMSC, Lampert JB, Stella RCR. Abordagens pedagógicas e tendências de mudanças nas escolas médicas. Rev Bras Educ Med. 2009; 33 Supl1:55-62.

18. Stewart M, Brown JB, Weston WW, McWhinney IR, McWilliam CL, Freeman TR. Pacient-centered medicine: transforming the clinical method. 2a ed. United Kingdom: Sage; 2003.

19. Ribeiro MMF, Amaral AFS. Medicina centrada no paciente e ensino médico: a importância do cuidado com a pessoa e o poder médico. Rev Bras Educ Med. 2008; 32(1):90-7.

20. Troncon LEA. Ensino clínico na comunidade. Medicina (Ribeirão Preto). 1999; 32(3):335-44.

21. Campos GWS. Papel da rede de atenção básica em saúde na formação médica diretrizes. Cad ABEM. 2007; 3:6-10.

22. Gomes R, Francisco AM, Tonhom SFR, Costa MCG, Hamamoto CG, Pinheiro OL, et al. A formação médica ancorada na aprendizagem baseada em problema: uma avaliação qualitativa. Interface (Botucatu). 2009; 13(28):71-83.

23. Mitre SM, Siqueira-Batista R, Girardi-de-Mendonça JM, Morais-Pinto M, Meirelles $C A B$, Pinto-Porto $C$, et al. Metodologias ativas de ensino-aprendizagem na formação profissional em saúde: debates atuais. Cienc Saude Colet. 2008; 13 Supl2:2133-44. 
24. Cezar PHN, Guimarães FT, Gomes AP, Rôças G, Siqueira-Batista R. Transição paradigmática na educação médica: um olhar construtivista dirigido à aprendizagem baseada em problemas. Rev Bras Educ Med. 2010; 34(2):298-303.

25. Feuerwerker LCM. Além do discurso de mudança na educação médica - processos e resultados. São Paulo: Hucitec; 2002.

26. Aréchiga H. Ciência e humanismo em medicina. México: Siglo XXI; 2003.

27. Cyrino EG, Toralles-Pereira ML. Trabalhando com estratégias de ensino-aprendizado por descoberta na área da saúde: a problematização e a aprendizagem baseada em problemas. Cad Saude Publica. 2004; 20(3):780-8.

28. Varga CRR, Almeida VC, Germano CMR, Melo DG, Chachá SGF, Souto BGA, et al. Relato de experiência: o uso de simulações no processo de ensino-aprendizagem em medicina. Rev Bras Educ Med. 2009; 33(2):291-7.

29. Aquilante AG, Silva RF, Avó LRS, Gonçalvez FGP, Souza MBB. Situações-problema simuladas: uma análise do processo de construção. Rev Bras Educ Med. 2011; 35(2):147-56.

30. Canuto AMM, Batista SHSS. Concepções do processo ensino-aprendizagem: um estudo com professores de medicina. Rev Bras Educ Med. 2009; 33(4):624-32.

31. Colliver JA. Effectiveness of problem-based learning curricula: research and theory. Acad Med. 2000; 75:259-66.

32. Almeida NG, Batista NA. Desempenho docente no contexto PBL: essência para aprendizagem e formação médica. Rev Bras Educ Med. 2013; 37(2):192-201.

33. Batista NA, Batista SHSS. A prática como eixo de aprendizagem na graduação médica. In: Puccini RF, Sampaio LO, Batista NA, organizadores. A formação médica na UNIFESP excelência e compromisso social. São Paulo: Unifesp; 2008. p. 101-15.

34. Silva RF, Aquilante AG, Zem-Mascarenhas SH, Kishi RGB, Varga CRR. Análise das situações simuladas da prática médica. Rev Bras Educ Med. 2012; 36(2):204-11.

35. Pazin Filho A, Scarpelini S. Simulação: definição. Medicina (Ribeirão Preto). 2007; 40(2):162-6.

36. Marin MJS, Lima EFG, Paviotti AB, Matsuyama DT, Silva LKD, Gonzalez C, et al. Aspectos das fortalezas e fragilidades no uso das metodologias ativas de aprendizagem. Rev Bras Educ Med. 2010; 34(1):13-20.

37. Pourshanazari A, Roohbakhsh A, Khazaei M, Tajadini H. Comparing the long-term retention of a physiology course for medical students with the traditional and problembased learning. Adv Health Sci Educ. 2013; 18(1):91-7.

38. Ausubel DP. Educational psychology: a cognitive view. New York: Holt, Rinehart and Winston; 1968.

39. Moreira MA, Masini EFS. Aprendizagem significativa - a teoria de David Ausubel. São Paulo: Centauro; 2002.

40. Gomes AP, Dias-Coelho UC, Cavalheiro PO, Gonçalvez CAN, Rôças G, Siqueira-Batista R. A educação médica entre mapas e âncoras: a aprendizagem significativa de David Ausubel, em busca da Arca Perdida? Rev Bras Educ Med. 2008; 32(1):105-11.

41. Almeida MJ, Campos JJB, Turini B, Nicoletto SCS, Pereira LA, Rezende LR, et al. Implantação das diretrizes curriculares nacionais na graduação em medicina no Paraná. Rev Bras Educ Med. 2007; 31(2):156-65.

42. Santos NR. SUS, política pública de Estado: seu desenvolvimento instituído e instituinte e a busca de saídas. Cienc Saude Colet. 2013; 18(1):273-80. 
43. Cunha GT, Campos GWS. Apoio matricial e atenção primária em saúde. Saude Soc. 2011; 20(4):961-70.

44. Lown B. A arte perdida de curar. Velloso W, tradutor. 3a ed. São Paulo: JSN Editora e Fundação Peirópolis; 1996.

45. Siqueira-Batista R, Gomes AP, Albuquerque VS, Cavalcanti FOL, Cotta RMM. Educação e competências para o SUS: é possível pensar alternativas à(s) lógica(s) do capitalismo tardio? Cienc Saude Colet. 2013; 18(1):159-70.

46. Campos GWS. Saúde Paidéia. 2a ed. São Paulo: Hucitec; 2005.

47. Lampert JB, Costa NMSC, Perim GL, Abdalla IG, Aguilar-da-Silva RH, Stella RCR. Tendências de mudanças em um grupo de escolas médicas brasileiras. Rev Bras Educ Med. 2009; 33 Supl1:19-34.

48. Ferreira RC, Fiorini VML, Crivelaro E. Formação profissional no SUS: o papel da atenção básica em saúde na perspectiva docente. Rev Bras Educ Med. 2010; 34(2):207-15.

49. Adler MS, Gallian DMC. Formação médica e serviço único de saúde: propostas e práticas descritas na literatura especializada. Rev Bras Educ Med. 2014; 38(3):388-96.

50. Azevedo CC, Ribeiro MAT, Batista SHSS. O humanismo na perspectiva de estudantes de medicina da UFAL. Rev Bras Educ Med. 2009; 33(4):586-94.

51. Gil CRR, Turini B, Cabrera MAS, Kohatsu M, Orquiza SMC. Interação ensino, serviços e comunidade: desafios e perspectivas de uma experiência de ensino-aprendizagem na atenção básica. Rev Bras Educ Med. 2008; $32(2): 230-9$.

52. Gomes AP, Rego S. Transformação da educação médica: é possível formar um novo médico a partir de mudanças no método de ensino-aprendizagem? Rev Bras Educ Med. 2011; 35(4):557-66.

53. Schraiber LB, Mota A. O social na saúde: trajetória e contribuições de Maria Cecília Ferro Donnangelo. Cienc Saude Colet. 2015; 20(5):1467-73.

54. Santos JS, Scarpelini S, Brasileiro SLL, Ferraz CA, Dallora MELV, Sá MFS. Avaliação do modelo de organização da unidade de emergência do HCFMRP-USP, adotando, como referência, as políticas nacionais de atenção. Medicina (Ribeirão Preto). 2003; 36(2/4):498-515.

55. Campos GWS, Guerrero AVP. Manual de práticas de atenção básica - saúde ampliada e compartilhada. São Paulo: Hucitec; 2008.

56. Benevides-Pereira AMT, Gonçalves MB. Transtornos emocionais e a formação em medicina: um estudo longitudinal. Rev Bras Educ Med. 2009; 33(1):10-23.

57. Rautio A, Sunnari $V$, Nuttinen $M$, Laitala M. Mistreatment of university students most common during medical studies. BMC Med Educ. 2005; 5:36. DOI: http://dx.doi.org/10.1186/1472-6920-5-36.

58. São Carlos Oficial. Santa Casa e UFSCar assinam convênio para Internato Médico dos estudantes de Medicina [Internet]. 2015 Fev [citado 25 Set 2015]. Disponível em: http://www.saocarlosoficial.com.br/ noticias $/ \mathrm{n}=$ Santa + Casa $+\mathrm{e}+$ UFSCar+assinam + convenio + para + Internato + Medico +dos + estudantes + de + Medicina_WIUILWJHEL.

59. Universidade Federal de São Carlos. Prefeitura Municipal de São Carlos e UFSCar firmam compromissos para finalização da transferência do Hospital Escola à Universidade [Internet]. 2015 [citado 25 Set 2015]. Disponível em: http://www.blogdareitoria.ufscar.br/?p=2341.

60. Abdelkhalek N, Hussein A, Gibbs T, Hamdy H. Using team-based learning to prepare medical students for future problem-based learning. Med Teach. 2010; 32(2):123-9.

61. Okubo Y, Ishiquro N, Suganuma T, Nishikawa T, Takubo T, Kojimahara N, et al. Team-based learning, a learning strategy for clinical reasoning, in students with problem-based learning tutorial experiences. Tohoku J Exp Med. 2012; 227(1):23-9.

62. Polyzois I, Claffey N, Mattheos N. Problem-based learning in academic health education. A systematic literature review. Eur J Dent Educ. 2010; 14(1):55-64.

63. Campos GWS, Amaral MP. A Clínica ampliada e compartilhada, a gestão democrática e redes de atenção como referenciais teórico-operacionais para a reforma do hospital. Cienc Saude Colet. 2007; 12(4):849-59. 
Adler MS, Gallian DMC. Escuela médica y Sistema Brasileño de Salud (SUS): creación de la escuela de medicina de la Universidad Federal de São Carlos, SP, Brasil (UFSCar) bajo la perspectiva de docentes y alumnos. Interface (Botucatu). 2018; 22(64):237-49.

La Universidad Federal de São Carlos (UFSCar) implantó su curso de Medicina en 2006, con un currículo basado en competencia y aprendizaje práctico integrado al SUS. El objetivo de este trabajo es presentar una encuesta con docentes y alumnos de graduación de la Primera Promoción de Medicina-UFSCar, realizada por medio de la Metodología de la Historia Oral de Vida. Las narrativas indican que el currículum favoreció el desarrollo de competencias tales como: capacidad de búsqueda, pensamiento crítico-reflexivo y autogestión del aprendizaje, a pesar de relatos sobre deficiencias en infraestructura. La práctica integrada al SUS, especialmente en las Unidades de Salud de la Familia, perfeccionó las dimensiones intelectivas, relacionales y afectivas del cuidado, favoreciendo la autonomía profesional.

Palabras clave: Enseñanza de graduación en medicina. Educación basada en competencias. Aprendizaje basado en problemas. Sistema Brasileño de Salud. 
